\title{
Different macrophages equally induce EMT in endometria of adenomyosis and normal
}

\author{
Min An¹, Dong Li ${ }^{2}$, Ming Yuan¹, Qiuju Li ${ }^{1}$, Lu Zhang ${ }^{1}$ and Guoyun Wang ${ }^{1}$ \\ ${ }^{1}$ Department of Obstetrics and Gynecology, Qilu Hospital of Shandong University, Jinan, People's Republic of \\ China and ${ }^{2}$ Cryomedicine Lab, Qilu Hospital of Shandong University, Jinan, People's Republic of China \\ Correspondence should be addressed to G Wang; Email: wangguoy@sdu.edu.cn
}

\begin{abstract}
Endometrial cells and microenvironment are two important factors in the pathogenesis of adenomyosis. Our previous study demonstrated that macrophages can induce eutopic epithelial cells of adenomyosis to suffer from epithelial-mesenchymal transition (EMT). The aim of this study is to detect whether macrophages interacting with epithelial cells equally induce the EMT process in normal and eutopic endometria of healthy and adenomyotic patients; and whether macrophages parallelly polarize to M2. We investigated the expression levels of epithelial cadherin (E-cadherin), neural cadherin ( $\mathrm{N}$-cadherin), cytokeratin7 (CK7), vimentin, transforming growth factor- $\beta 1$ (TGFB1), SMAD3 and pSMAD3 using immunohistochemistry and western blot, and then estimated the genetic levels of $C D 163, I L 10$ and MMP12 using real-time quantitative polymerase chain reaction (RT-PCR) in macrophages. Eutopic and normal endometrial tissues were obtained from 20 patients with adenomyosis and 11 control patients without adenomyosis, respectively. The immunohistochemical analysis shows distinct EMT in eutopic endometria in secretory phase; the expression levels of TGFB1, SMAD3 and pSMAD3 that indicate signal pathway of EMT were also higher in secretory phase. Macrophages can induce EMT process in primary endometrial epithelial cells derived from normal and eutopic endometria. After co-culturing, THP-1-derived macrophages polarized to M2. Compared with the eutopic endometrium group, further polarization to M2 was observed in the normal endometrium group. These results indicate that adenomyosis may be promoted by the pathologic EMT of epithelial cells, which is induced by macrophages that incapably polarize to M2.

Reproduction (2017) 154 79-92
\end{abstract}

\section{Introduction}

Adenomyosis, an estrogen-dependent inflammatory disease, is defined as the presence of endometrial glands and stroma deep within the myometrium; two distinct forms of adenomyosis, namely, diffuse and focal, have been described (Ferenczy 1998). The main clinical features of adenomyosis are dysmenorrhea and menorrhagia, which are significantly associated with peritoneal endometriosis in infertile patients at reproductive age (Kunz et al. 2000, 2005). However the pathogenesis of adenomyosis remains unclear. The only difference between adenomyosis and endometriosis is the site of endometriotic tissues, that is, inside or outside the uterus (Ota et al. 1998). Thus, knowledge on the endometriotic cell origin is indispensable for the development of preventive and targeted treatment strategies for adenomyosis. The most widely accepted theory on the pathogenesis of adenomyosis is the downgrowth and invagination of the endometrium into the myometrium (Bergeron et al. 2006); however the possible mechanism of gland invagination from the endometrium deep into the underlying myometrium is unknown.
Epithelial-mesenchymal transition (EMT) and its converse, mesenchymal-epithelial transition (MET), were defined decades ago (Alcorn et al. 1999). Once epithelial cells become competent to respond to EMT-inducing signals, these signals can promote the disruption of the intercellular adhesion complexes and the loss of the apicobasal polarity of the epithelial cells, a prime feature crucial for cells to leave the epithelium and achieve migration potentiality (Khan et al. 2015). Many studies indicated that EMT is a crucial process in adenomyosis and endometriosis lesions (Chen et al. 2010, Matsuzaki \& Darcha 2012), and the endometrium and inner myometrium are closely apposed without any intervening basement membrane; thus, EMT events might occur here.

A hallmark of EMT is the downregulation of epithelial cadherin (E-cadherin) to reinforce the destabilization of adherens junctions. Specifically, the downregulation of E-cadherin is balanced by the increased expression of mesenchymal neural cadherin ( $\mathrm{N}$-cadherin), which results in a 'cadherin switch' that alters cell adhesion (Wheelock et al. 2008). Alterations in the expression of genes encoding cytoskeletal and polarity complex 
proteins also contribute to EMT. Keratin and vimentin filaments regulate the trafficking of organelles and membrane-associated proteins, but show differences in the proteins that they target to the membrane (Lamouille et al. 2014). Thus, N-cadherin, E-cadherin, vimentin and cytokeratin7 (CK7) are markers for EMT and play different roles during the EMT process.

Macrophages have been known to play important roles in the adenomyosis process (Ota et al. 1998). One study reported an increased stromal macrophage population in the functional layer of the endometrium in patients experiencing diffuse and focal adenomyosis (Tremellen \& Russell 2012). Another study indicated that, after treating adenomyosis patients with the gonadotropinreleasing hormone $(\mathrm{GnRH})$ agonist, the infiltration of CD68-positive macrophages is significantly decreased in the endometrium of adenomyotic women (Khan et al. 2010). Although macrophages have been known to be involved in the adenomyosis process by enhancing cell growth and proliferation through the amplification of cytokine secretion (Chen et al. 2003, Shao et al. 2016), the number and identity of factors that act on this regulatory process are still unknown.

Abnormal levels of macrophages, which are important components of immune cells, have been largely reported in adenomyosis (Ota et al. 1998, Zhihong et al. 2016). Increased knowledge on the immune aspects of the pathogenesis of adenomyosis is needed for this debilitating condition. To our knowledge, macrophages, which play important roles in innate and acquired immunity, together with natural killer cells and cytotoxic T-lymphocytes in healthy women, can destroy misplaced endometrial cells (Dmowski et al. 1998). The EMT process induced by macrophages has been investigated systematically in tissue repair, remodeling, fibrosis (Scotton \& Chambers 2007), and tumor progression (Galdiero et al. 2013). Alternatively activated (M2) macrophages are the major type associated with the tumor EMT process. Like tumor progression, adenomyosis also exhibits the EMT process.

Our previous study showed that THP-1-derived macrophages induced EMT process in endometrial epithelial cells of patients with adenomyosis (Min et al. 2017). A recent study involving EMT of endometrial epithelial cells was performed on human primary endometrial epithelial cells, which were isolated from normal endometrial tissues (Xiong et al. 2016). Therefore we want to investigate if the macrophages can induce normal epithelial cells to undergo EMT, and if macrophages interacting with epithelial cells equally induce the EMT process in normal and eutopic endometria of healthy and adenomyotic patients. Also, in our previous study (Min et al. 2017), after co-culturing with eutopic epithelial cells of adenomyosis, THP-1derived macrophages polarized to M2. Thus, we also want to estimate if the macrophages parallelly polarize to $\mathrm{M} 2$ between eutopic endometrium and normal endometrium.

Given that macrophages and EMT are all involved in the adenomyosis process, and the definite mechanism by which macrophages promote the development of adenomyosis is vague, macrophages are presumed to induce endometrial epithelial cells to undergo EMT because macrophages can induce EMT in many other diseases. The co-culture system was used in this study to test the EMT process of epithelial cells isolated from normal endometrium and adenomyotic eutopic endometrium induced by macrophages derived from THP-1. As a predominant signaling pathway in EMT, the protein expression levels of transforming growth factor- $\beta 1$ (TGFB1) and SMAD3/pSMAD3 in epithelial cells were also evaluated. The macrophages were simultaneously collected to estimate the gene expression levels of CD163, IL10 and MMP12, a group of classic markers for M2 in the co-culture system.

\section{Materials and methods \\ Ethical approval}

The study protocol was approved by the Institutional Review Board of the Qilu Hospital Authority (KYLL-2015-077).

\section{Patients and tissue samples}

This study recruited 20 women with adenomyosis who were diagnosed by ultrasound doctors before operations and clinical pathologists after hysterectomy at the Qilu Hospital of Shandong University from June 2015 to March 2016. After obtaining the patients' written informed consent, eutopic endometrium tissues were collected during the operation and immediately sent to the laboratory. The tissues were separated into two parts: one was cultured in vitro, and the remaining was stored with $10 \%$ buffered formalin and processed for paraffin embedding. For the controls, endometrial tissue samples were collected through curettage from 11 women who exhibited fallopian tube jam but without any clinical indication or history of adenomyosis or endometriosis. The diagnosis was done by ultrasound doctors before operations, and then doctors and clinical pathologists made a definite diagnosis after laparoscopic surgery. The characteristics of patients recruited with adenomyosis and controls are shown in Table 1. Because four patients with adenomyosis had endometrial polyps, they were excluded for the present analysis.

\section{Immunohistochemical (IHC) staining}

The slides were subjected to immunohistochemical (IHC) analysis. Tissue sections were dewaxed and rehydrated in ethanol and water. Antigen retrieval was performed in citrate buffer ( $\mathrm{pH} 6.0,15 \mathrm{~min}$ ), and endogenous peroxidase activity was eliminated by incubation in $3 \%$ hydrogen peroxide. The tissue sections incubated overnight at $4^{\circ} \mathrm{C}$ for rabbit primary antibody against human CK7 (ab68459, Abcam, 1:400), 
Table 1 Characteristics of patients recruited with adenomyosis and controls.

\begin{tabular}{|c|c|c|c|}
\hline Item & Controls $(n=11)$ & Adenomyosis $(n=20)$ & Statistical significance \\
\hline Age (year) & & & $* *$ \\
\hline Mean (S.D.) & $30.0(8.7)$ & $40.8(5.1)$ & \\
\hline Median (range) & $30(20-41)$ & $40.5(31-49)$ & \\
\hline Menstrual phase & & & NS \\
\hline Proliferative & $7(63.6 \%)$ & $9(45.0 \%)$ & \\
\hline Secretory & $4(36.4 \%)$ & $11(55.0 \%)$ & \\
\hline Gravidity & & & $* * *$ \\
\hline 0 & $3(27.3 \%)$ & $1(5.0 \%)$ & \\
\hline 1 & $4(36.4 \%)$ & $2(10.0 \%)$ & \\
\hline$\geq 2$ & $4(36.4 \%)$ & $17(85.0 \%)$ & \\
\hline Abortion & & & $* * *$ \\
\hline 0 & $4(36.3 \%)$ & $4(20.0 \%)$ & \\
\hline 1 & $5(45.5 \%)$ & $3(15.0 \%)$ & \\
\hline$\geq 2$ & $2(18.2 \%)$ & $13(65.0 \%)$ & \\
\hline $\begin{array}{l}\text { Visual analog scale on the severity of } \\
\text { dysmenorrhea }\end{array}$ & & & $* * *$ \\
\hline Mean (S.D.) & $0.5(1.1)$ & $5.9(2.3)$ & \\
\hline Median (range) & $0(0-5)$ & $6(0-8)$ & \\
\hline Uterus size $\left(\mathrm{cm}^{3}\right)$ & & & $* * *$ \\
\hline Mean & 72.5 & 344.3 & \\
\hline Median (range) & $70.1(52.3-80.1)$ & $285.5(110.1-628.2)$ & \\
\hline Co-occurrence of endometriosis & & & NS \\
\hline No & $11(100.0 \%)$ & $18(90.0 \%)$ & \\
\hline Yes & $0(0.0 \%)$ & $2(10.0 \%)$ & \\
\hline Co-occurrence of endometrial polyps & & & NS \\
\hline No & $11(100.0 \%)$ & $16(80.0 \%)$ & \\
\hline Yes & $0(0.0 \%)$ & $4(20.0 \%)$ & \\
\hline
\end{tabular}

VAS, visual analog scale; uterus size calculated as $\pi \mathrm{D} 1 \mathrm{D} 2 \mathrm{D} 3 / 6$, where $\mathrm{D} 1=$ the distance from fundus to the internal OS of the cervix, $\mathrm{D} 2=$ transverse diameter at the level of the cornua and D3 = anteroposterior diameter at the level of cornua. ${ }^{*} P<0.05 ;{ }^{* * P} P<0.01 ;{ }^{* * *} P<0.001$; NS: $P>0.05$. Wilcoxon's rank test was used for age, VAS and uterus size while for other data Fisher's exact test was used.

E-cadherin (ab40772, Abcam, 1:400), N-cadherin (ab18203, Abcam, 1:400), vimentin (ab92547, Abcam, 1:700), TGFB1 monoclonal antibody (ab92486, Abcam, 1:200), SMAD3 monoclonal antibody (ab40854, Abcam, 1:200), and pSMAD3 monoclonal antibody (ab52903, Abcam, 1:100). The secondary antibody kit (CWBIO, Beijing, China) was utilized to link the primary antibody. The sections were counterstained with hematoxylin, dehydrated in ethanol and xylene, and mounted in Permount mounting medium. The immunostaining results were evaluated using a previously reported method (Shen et al. 2015). The images were obtained using a microscope (Olympus BX53, Olympus), which was fitted with a digital camera (Olympus cellSens Standard, Olympus). A series of five images was randomly selected from several sections per tissue sample. Each image was taken for each immunostained marker to yield a mean optional density value by Image Pro-Plus 6.0 (Media Cybernetics, Inc., Bethesda, MD, USA). Staining was defined via color intensity, and a color mask was made. The mask was then applied equally to all images, and subsequent measurement readings were obtained. Immunohistochemical parameters assessed in the area detected included (1) integrated optical density (IOD), (2) total stained area (S) and (3) the mean optical density (MOD), equivalent to the mean intensity of staining across all glands.

\section{Primary endometrial cell culture}

The isolation and culture of adenomyotic primary eutopic endometrial cells and normal endometrial cells were conducted using a previously reported method (Chan et al. 2004). The endometrial tissues were minced into small pieces $\left(1 \mathrm{~mm}^{3}\right)$. After the minced tissues were subjected to enzymatic digestion with $0.25 \%(\mathrm{w} / \mathrm{v})$ collagenase II mixed with $0.125 \%(\mathrm{w} / \mathrm{v})$ collagenase IV (Worthington, Lakewood, $\mathrm{NJ}$, USA) for $75 \mathrm{~min}$ at $37^{\circ} \mathrm{C}$, the tissues were filtered initially through a $100 \mu \mathrm{m}$ (pore size) nylon mesh to remove debris and then through a $40 \mu \mathrm{m}$ (pore size) nylon mesh (Falcon cell strainers; Thermo Fisher Scientific). The epithelial cells remaining in the cell strainer were collected, resuspended in Dulbecco's modified Eagle's medium/F12 (Gibco), and plated onto six-well plates. Then, the culture medium was changed to RPMI-1640 medium (Gibco) to prepare for co-culturing with macrophages. The endometrial cells were observed through an inverted microscope, and the cellular morphology of epithelial cells is shown in Fig. 1A. The endometrial cells were verified through immunofluorescent staining by using an antibody against CK7 (ab68459, Abcam, 1:200), which is a specific marker for epithelial cells (Fig. 1B). The purity of epithelial cells isolated from endometria was greater than 95\%, which was in conformity with a previous study (Kao et al. 2011).

\section{Epithelial cells co-cultured with macrophages}

THP-1 cells (acute monocytic leukemia) were provided by Dr Chengjiang Gao (Shandong University, School of Medicine, Department of Immunology, Jinan, Shandong, China). Macrophage differentiation of the THP-1 cells was conducted using a previously reported method (Dehai et al. 2014). 


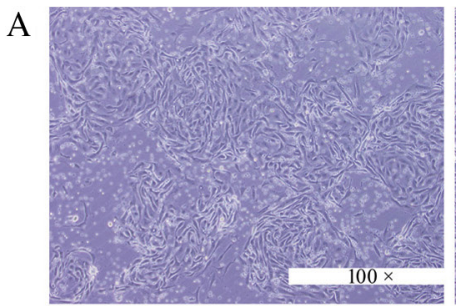

eutopic epithelial cells

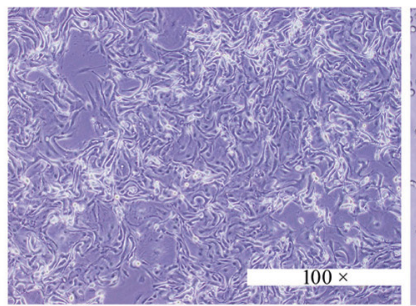

normal epithelial cells

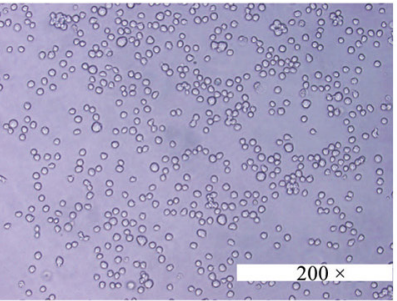

THP-1 cells

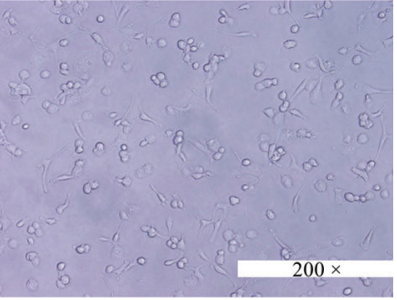

THP-1 cells treated with PMA

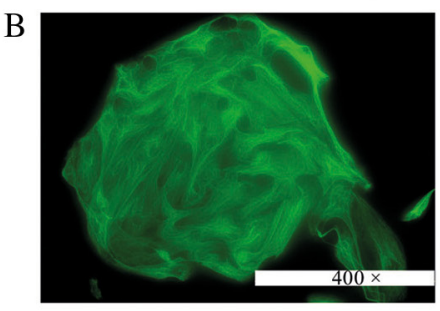

CK7

$\mathrm{C}$

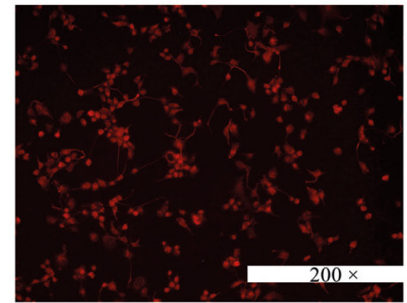

CD68

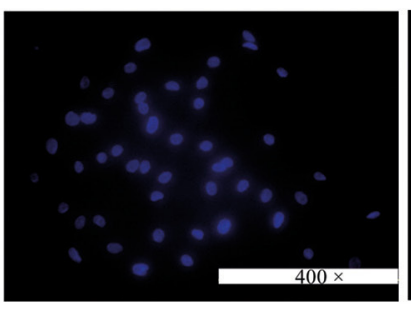

DAPI

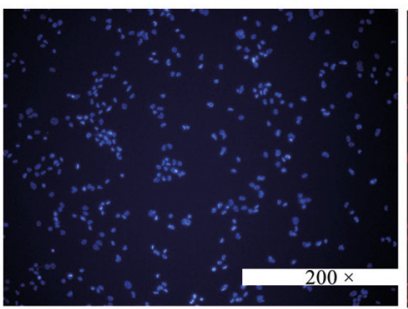

DAPI

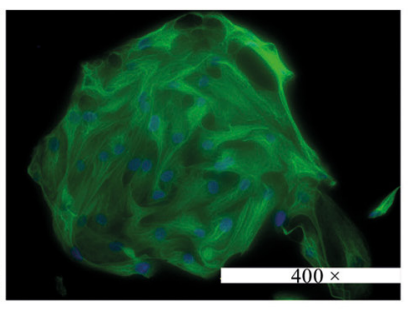

merge

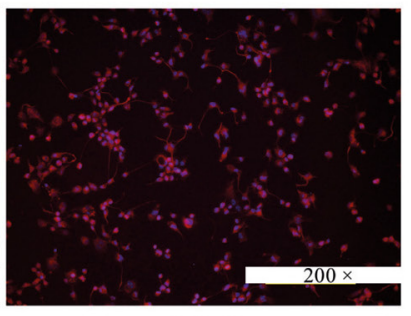

merge
$\mathrm{D}$

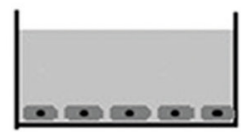

primary epithelial cells

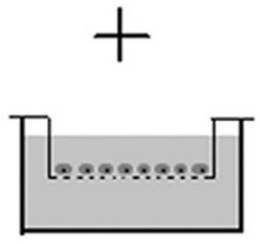

macrophages derived from THP-1

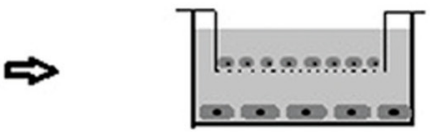

co-cultured system

Figure 1 (A) Isolated and cultured primary epithelial cells as well as THP-1 cells cultured with or without PMA. (B) Epithelial cells isolated from endometrium were verified by immunofluorescent staining (nonlinear adjustment). CK7, stained in green, was used as the marker for epithelia. DAPI, stained in dark blue, was used as the marker for cell nuclei. (C) Macrophages derived from THP-1 cells were verified by

immunofluorescent staining (nonlinear adjustment). CD68, stained in red, was used as the marker for macrophages. DAPI, stained in dark blue, was used as the marker for cell nuclei. (D) The sketch map of the co-culturing system.

The cells were triggered by adding $100 \mathrm{ng} / \mathrm{mL}$ phorbol 12-myristate 13-acetate (PMA; Sigma-Aldrich) for two days and washed thrice with phosphate-buffered saline (PBS) to eliminate the effect of PMA. Macrophages were observed through an inverted microscope, and the cellular morphology is shown in Fig. 1A. The macrophages were also verified through immunofluorescent staining using an antibody against CD68 (ab955, 1:200; Abcam), which is a marker for macrophages (Fig. 1C). The primary endometrial epithelial cells were co-cultured with THP-1-derived macrophages using a standard Transwell insert $(0.4 \mu \mathrm{m}$; Corning) (Fig. 1D). Each well was plated with approximately 10,000 primary endometrial cells. The cells were washed after incubation for $24 \mathrm{~h}$ with $10 \%$ fetal bovine serum medium, and the inserts with induced macrophages $\left(7.5 \times 10^{5}\right.$ cells $)$ were added to the wells. The control group had an empty insert. Epithelial cells were harvested at different time points, that is, on days 1, 2, 4 and 6. 


\section{Immunocytochemistry}

We performed immunocytochemistry staining to show morphological changes of epithelial cells with EMT-related proteins as previously described (Xue et al. 2013). The cultured epithelial cells were washed twice with PBS and fixed in 4\% paraformaldehyde ( $\mathrm{pH}$ 7.0) for $30 \mathrm{~min}$. The cells were then washed in PBS, blocked with $10 \%$ normal goat serum for $1 \mathrm{~h}$, and incubated overnight with rabbit anti-human CK7 monoclonal antibody (ab68459, 1:400; Abcam) and rabbit anti-human vimentin monoclonal antibody (ab92547, 1:600; Abcam) at $4{ }^{\circ} \mathrm{C}$. The secondary antibody kit (CWBIO, Beijing, China) was utilized to link the primary antibody. Cells were counterstained with hematoxylin before mounting.

\section{Western blot}

The EMT-like process-related proteins were examined using western blot analysis for primary endometrial cells at four different time points after co-culturing the cells with THP-1derived macrophages. The cells were scraped and extracted in a commercial kit (BestBio, Shanghai, China) for total protein extraction. All proteins that were mixed with sodium dodecyl sulfate-polyacrylamide gel electrophoresis (SDSPAGE) loading buffer (P0015; Beyotime, Shanghai, China) were heated for $5 \mathrm{~min}$ at $100^{\circ} \mathrm{C}$. Protein samples were loaded onto $10 \%$ SDS-PAGE and electroplated onto polyvinylidene fluoride membranes (Millipore). The membranes were blocked in 5\% nonfat milk (BD, Franklin Lakes, NJ, USA) reconstituted in TBST $(0.15 \mathrm{M} \mathrm{NaCl}, 0.05 \%$ Tween 20 , and $10 \mathrm{mM}$ Tris- $\mathrm{HCl}$ ( $\mathrm{pH} 8.0)$ ) for $70 \mathrm{~min}$ at room temperature and subsequently incubated overnight at $4{ }^{\circ} \mathrm{C}$ with the following primary antibodies: rabbit anti-human CK7 monoclonal antibody (ab68459, 1:5,000; Abcam), rabbit anti-human E-cadherin monoclonal antibody (ab40772, 1:10,000; Abcam), rabbit anti-human vimentin monoclonal antibody (ab92547, 1:5000; Abcam), rabbit anti-human $\mathrm{N}$-cadherin monoclonal antibody (ab18203, 1:1000; Abcam), rabbit anti-human TGFB1 monoclonal antibody (ab92486, 1:1000; Abcam), rabbit anti-human SMAD3 monoclonal antibody (ab40854, 1:1000; Abcam), and rabbit anti-human GAPDH polyclonal antibody (10494-1-AP, 1:20,000; Proteintech, Wuhan, China). After incubating the membranes with horseradish-peroxidaselabeled secondary antibodies for $70 \mathrm{~min}$ at room temperature, the signal was detected by Image Studio Digits version 4.0. Three independent experiments were performed.

\section{Real-time polymerase chain reaction (RT-PCR)}

The co-cultured cells (macrophages) were washed with cold PBS, and the total RNA was extracted with TRIzol Reagent (Invitrogen Life Technologies) in accordance with the manual of the product owner. RNA $(1 \mu \mathrm{g})$ was reverse-transcribed into cDNA with ReverTra Ace quantitative PCR (qPCR) RT Master Mix with gDNA Remover (Code No. FSQ-301; Toyobo, Osaka, Japan). Each $20 \mu \mathrm{L}$ of PCR product contained $1 \times$ SYBR Green PCR Master Mix (Toyobo), $30 \mathrm{ng}$ of cDNA, and $300 \mathrm{nM}$ of each specific primer. The primers used for each gene are listed in Table 2. Subsequently, qPCR was performed on an Applied
Table 2 Primer sequences of each gene detected in real-time RT-PCR.

\begin{tabular}{lll}
\hline CD163 & Forward & GGCTTGCAGTTTCCTCAAGA \\
CD163 & Reverse & AGCTGACTCATGGGAATTTTCTG \\
MMP12 & Forward & ACTACACATTCAGGAGGCACA \\
MMP12 & Reverse & GTCATCAGCAGAGAGGCGAA \\
IL10 & Forward & AGGACTTTAAGGGTTACCTGGG \\
IL10 & Reverse & TTCTCAGCTTGGGGCATCAC \\
GAPDH & Forward & GCACCGTCAAGGCTGAGAAC \\
GAPDH & Reverse & TGGTGAAGACGCCAGTGGA \\
\hline
\end{tabular}

Biosystems 7500 RT-PCR System (Applied Biosystems). Three separate experiments were performed on different cultures, and each sample was assayed in triplicate. The mean was obtained to determine the mRNA levels by quantitative RT-PCR analysis, which was performed using the ABI 7500 RT-PCR system (Applied Biosystems). The gene expression levels for each group were normalized to glyceraldehyde 3-phosphate dehydrogenase $(\mathrm{GAPDH})$. The mean relative gene expression level was determined, and the differences were calculated using the $2^{-\Delta \Delta C t}$ method.

\section{Statistical analysis}

Graphical and statistical analyses were performed using the GraphPad prism software. Comparative statistical analyses were performed using the Mann-Whitney test of immunohistochemical staining. And Wilcoxon matched pairs test was performed of the antigen expression in western blot between the study (co-cultured with macrophage) and control groups; between the normal endometrium and eutopic endometrium groups, the Mann-Whitney test was used. The gene expression levels of macrophages among the control, normal endometrium and eutopic endometrium groups were calculated using the one-way analysis of variance (NewmanKeuls multiple comparison test). $P$ values $\leq 0.05$ were considered significant.

\section{Results}

\section{The different expressions of EMT-associated proteins in the normal and eutopic endometrial epithelial cells during menstrual}

To investigate whether EMT process exists in adenomyotic eutopic endometrium, we compared the expression levels of CK7, vimentin, E-cadherin and $\mathrm{N}$-cadherin in the endometrium with or without adenomyosis. As depicted in Figs 2 and 3, we found that the expression levels of $\mathrm{CK} 7, \mathrm{E}$-cadherin, $\mathrm{N}$-cadherin, TGFB1, SMAD3 and pSMAD3 have no significant difference between normal and eutopic endometrial epithelial cells in proliferative phase except vimentin, which was upregulated. While in the secretory phase, the expression levels of $\mathrm{CK} 7$ and E-cadherin were both downregulated in eutopic endometrial epithelial cells compared with the normal; as for vimentin, $\mathrm{N}$-cadherin, TGFB1 and SMAD3, the expression levels were both 


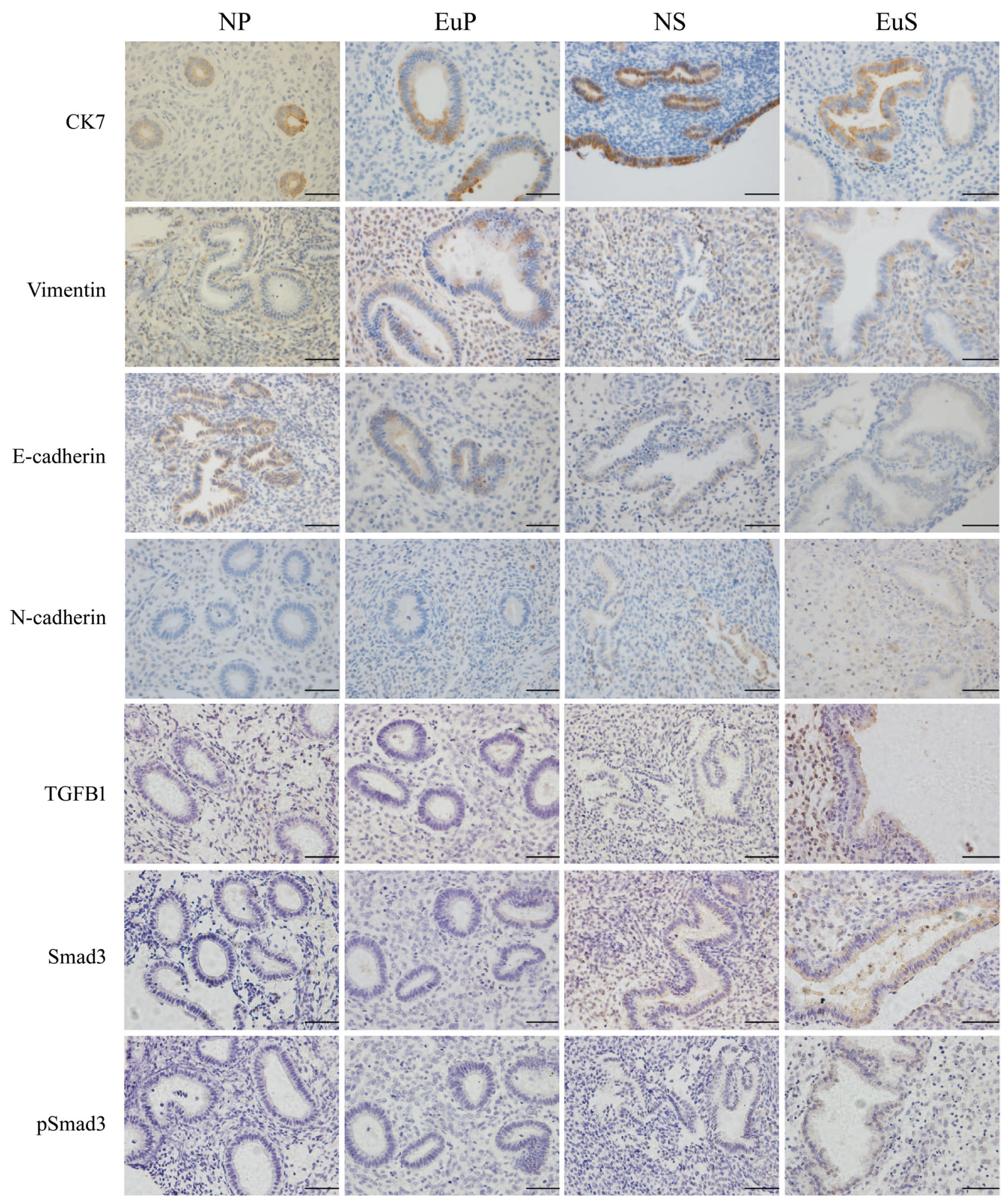

Figure 2 The expression levels of CK7, vimentin, E-cadherin, N-cadherin and several signal pathway proteins (TGFB1, SMAD3 and pSMAD3) in the endometrium of women without adenomyosis and endometriosis $(n=11)$, and eutopic endometrium $(n=16)$ of women with adenomyosis were analyzed by immunohistochemistry. Original magnification, $\times 400$; NP, endometrium of women without adenomyosis at proliferative phase; EuP, endometrium of patient with adenomyosis at proliferative phase; NS, endometrium of women without adenomyosis at secretory phase; EuS, endometrium of patient with adenomyosis at secretory phase. 


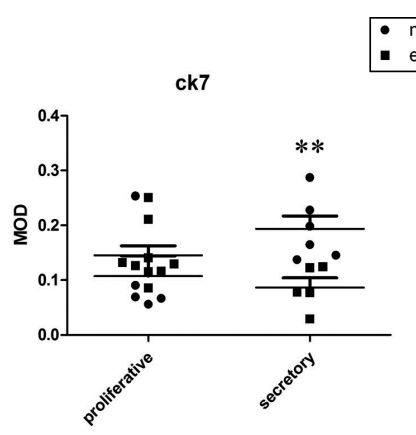

- normal

Vimentin
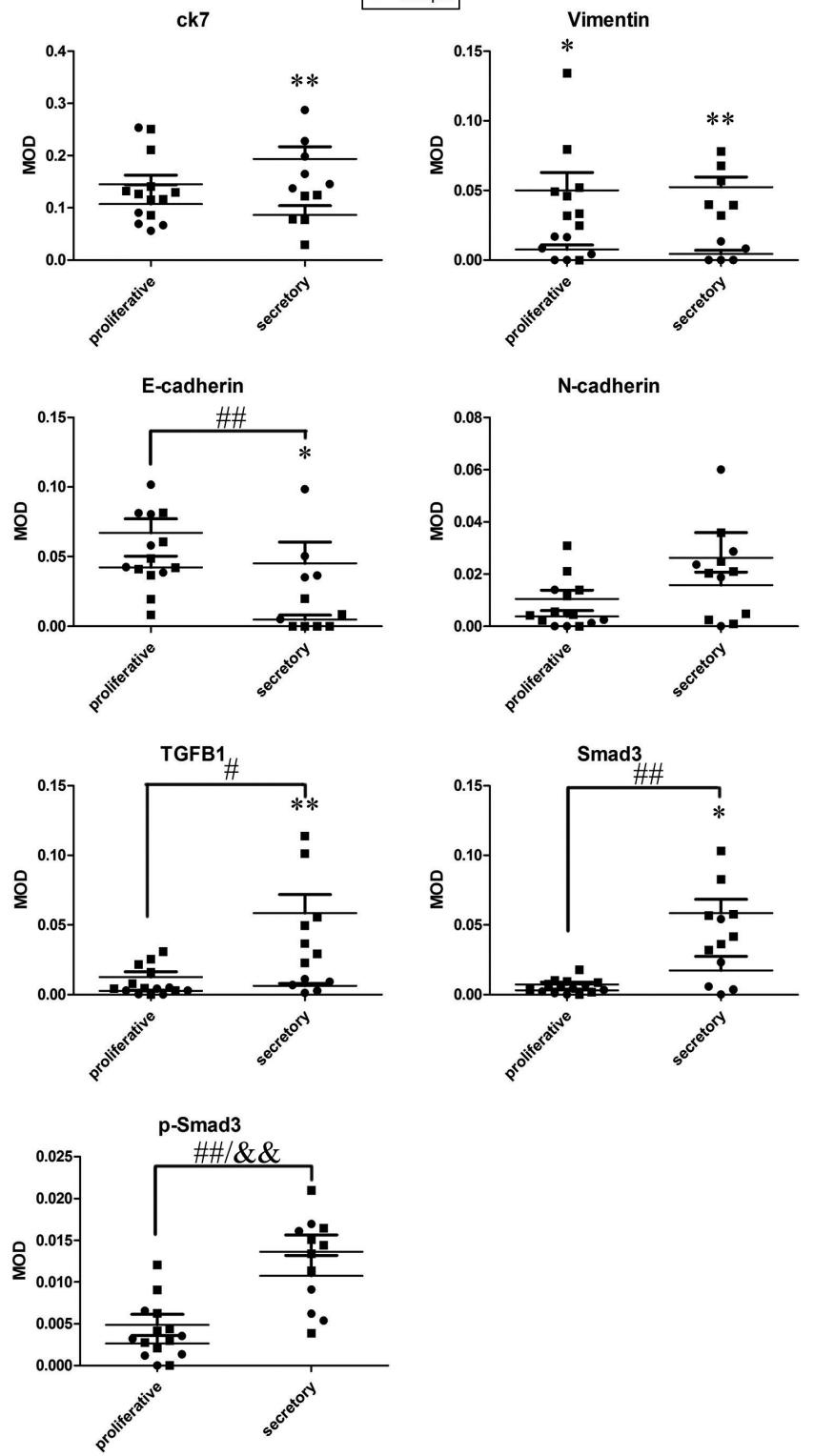

Figure 3 Results of MOD analysis (Mann-Whitney test) using all immunostaining data. All patients were represented by points. *Normal group vs eutopic group; ${ }^{*}$ proliferative phase (eutopic) vs secretory phase (eutopic); ${ }^{\&}$ proliferative phase (normal) vs secretory

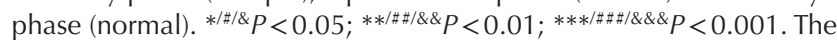
results are expressed as the mean \pm S.E.M.

high in secretory phase; the expression of pSMAD3 did not differ between eutopic and normal epithelial cells. We also compared the expression level of CK7, vimentin, E-cadherin, $\mathrm{N}$-cadherin, TGFB1, SMAD3 and pSMAD3 between proliferative and secretory phase. As shown in Figs 2 and 3, in eutopic endometrium, the expression levels of TGFB1, SMAD3 and pSMAD3 in secretory phase were higher than those proliferative phase; while in normal endometrium, only pSMAD3 was upregulated; however the expression of E-cadherin downregulated in secretory phase.

\section{Mesenchymal-like morphological changes of epithelial cells in immunocytochemistry staining after co-culture}

Immunocytochemical analyses of CK7 and vimentin expression were performed to investigate the morphological changes of epithelial cells. As shown in Fig. 4, analysis using an ordinary light microscope revealed mesenchymal-like changes in epithelial cells after they were co-cultured with macrophages for 2 days. The expression level of CK7 was downregulated and vimentin was upregulated in epithelial cells. Although the changes of cell morphology were less obvious than protein expression, some epithelial cells have typical mesenchymal-like morphology.

THP-1-derived macrophages induce primary endometrial epithelial cells to undergo EMT, and compared with the normal endometrium group, the downregulation of E-cadherin is evident in the eutopic endometrium group

Co-culturing of endometrial epithelial cells and macrophages can induce endometrial epithelial cells of the adenomyotic eutopic endometrium and normal endometrium to exhibit EMT, which was indicated by the downregulation of epithelial markers (E-cadherin and CK7) and the upregulation of mesenchymal markers (vimentin and $\mathrm{N}$-cadherin). After co-culturing with macrophages, the epithelial cells derived from the eutopic endometrium of adenomyosis patients exhibited EMT (Fig. 5A). Statistical analysis showed that, although the protein expression levels of CK7 and E-cadherin were downregulated during the entire experimental period, statistical significance was only observed on days 1, 4 and 6 (Fig. 5B). Moreover, the protein expression level of vimentin was significantly upregulated on day 6 (Fig. 5B). Similarly, EMT occurred in epithelial cells isolated from the normal endometrium (Fig. 5A). The time point that showed statistical significance was day 6 for vimentin expression (Fig. 5C). The protein expression level of control group, epithelial cells isolated from adenomyotic eutopic and normal endometria, was estimated first before comparing the variance $(\mathrm{m} / \mathrm{c}$; shown in Fig. 5A) of the protein expression of EMT between adenomyotic eutopic and normal endometria after co-culture with macrophages. Low expression of E-cadherin was observed in adenomyotic eutopic epithelial cells compared with the normal epithelial cells before co-culturing (Fig. 5D). After co-culturing with macrophages, there was no significance of the fold changes for $\mathrm{CK} 7, \mathrm{E}$-cadherin, vimentin and $\mathrm{N}$-cadherin expressions between the eutopic endometrium group and the normal endometrium group (Fig. 5E). 


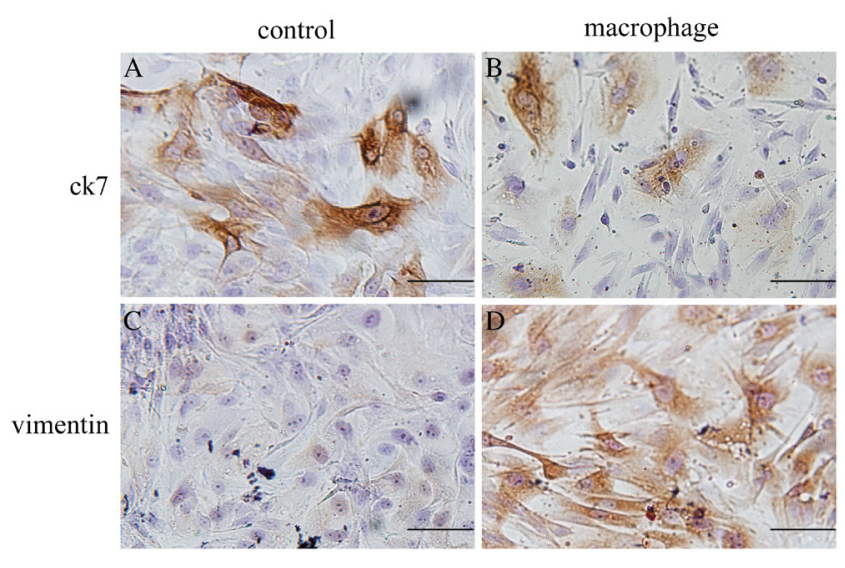

Figure 4 Immunocytochemical staining for vimentin and CK7 expression. The cytoplasm of cells was negative for vimentin (C) and positive for CK7 (A) before CO-culture; after 2 days of co-culture, the cytoplasm of cells was positive for vimentin (D) and negative for CK7 (B). The nuclei were stained with hematoxylin. Magnification, $\times 400$.

\section{Primary endometrial epithelial cells co-cultured with macrophages did not significantly enhance the TGFB1/SMAD3 protein expression level; however the expression level of pSMAD3 was upregulated}

The protein expression levels of TGFB1 and SMAD3 in the eutopic endometrium and normal endometrium groups were insignificantly upregulated after co-culturing endometrial epithelial cells and macrophages (Fig. 6A, $B$, and $C$ ). The protein expression level of control group epithelial cells isolated from adenomyosis eutopic and normal endometria was also estimated. Statistical analysis indicated no difference in the protein expression levels of TGFB1 and SMAD3 between adenomyosis eutopic and normal endometrial epithelial cells without co-culture (Fig. 6D). However the upregulation of pSMAD3 after co-culturing was higher on day 6 in the epithelial cells both derived from normal and eutopic endometria after co-culture (Fig. 6A, B and C); statistical analysis indicated that the protein expression levels of pSMAD3 in eutopic epithelial cells was higher than normal (Fig. 6D). After co-culturing with macrophages, the fold changes of TGFB1, SMAD3 and pSMAD3 have no difference between the eutopic endometrium group and the normal endometrium group (Fig. 6E).

\section{When co-cultured with endometrial cells, THP-1- derived macrophages polarized to the M2 type; compared with the eutopic endometrium group, further polarization to M2 was detected in the normal endometrium group}

The interaction of macrophages and endometrial epithelial cells induced the endometrial epithelial cells to undergo mesenchymal transition-like process. Meanwhile, endometrial epithelial cells can induce macrophages to polarize. The PCR results proved that primary endometrial epithelial cells can cause macrophages to polarize to the M2 type. The genetic level of ILIO in the eutopic endometrium and normal endometrium groups significantly increased when compared with the control group. The expression of IL 10 was higher in the normal endometrium group on day 1 and day 4 (Fig. 7B). However the genetic level of CD163 increased only in the normal endometrium group on day 1 and day 6 . The eutopic endometrium group did not exhibit CD163 expression in macrophages compared with the control group. Therefore in terms of induced CD163, the normal endometrium group exhibited higher levels than the eutopic endometrium group on day 1 and day 6 (Fig. 7B). The genetic level of MMP12 increased in the normal endometrium group at all the time points. In contrast to the control group, the MMP12 levels increased significantly in the eutopic endometrium group only on day 1 and day 2. The induction of MMP12 expression was higher in the normal endometrium group than in the eutopic endometrium group, except on day 2 (Fig. 7B). Throughout the experiment, the polarization of macrophages in the eutopic endometrium group was tender, whereas that in the normal endometrium group was fluctuant: the expression levels of CD163, IL10 and MMP12 initially increased, then decreased, and increased again. Although the polarization of macrophages was definite, the morphological change of macrophages was not evident (Fig. 7A).

\section{Discussion}

The EMT process shown in eutopic endometrial epithelial cells was comparable with that of the normal epithelial cells in IHC analysis at secretory phase; however it was not obvious in proliferative phase. The primary epithelial cells derived from adenomyotic eutopic and normal endometria were successfully induced to undergo EMT by co-culturing them with macrophages. In the co-cultured system, the macrophages derived from THP-1 polarized to $M 2$, and the polarization of macrophages was more intense in the normal endometrium group than in the eutopic endometrium group.

Eutopic endometrium has always been considered the origin of ectopic endometrium in endometriosis or adenomyosis (Benagiano et al. 2013). Research on the adenomyosis mechanism gained more attention after the following definition was provided (Bird et al. 1972): 'Adenomyosis may be defined as the benign invasion of endometrium into the myometrium, producing a diffusely enlarged uterus which microscopically exhibits ectopic non-neoplastic, endometrial glands and stroma surrounded by the hypertrophic and hyperplastic myometrium.' However the specific molecular mechanism is still unclear. In recent years, EMT has emerged in adenomyosis mechanism research. Although these studies had far-reaching significance, 
A

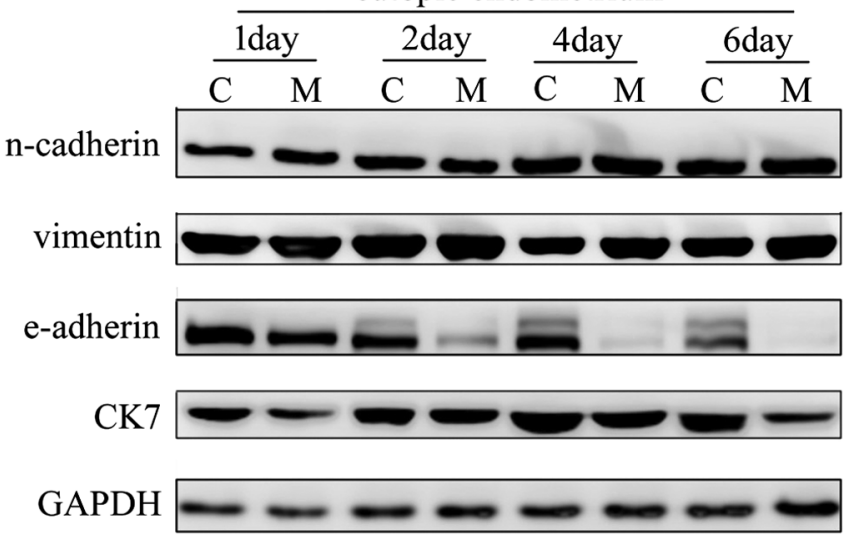

B
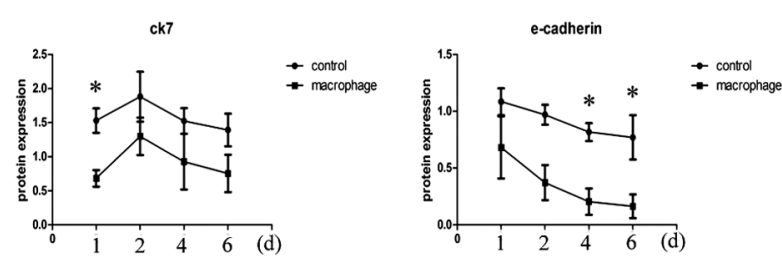

$\mathrm{C}$
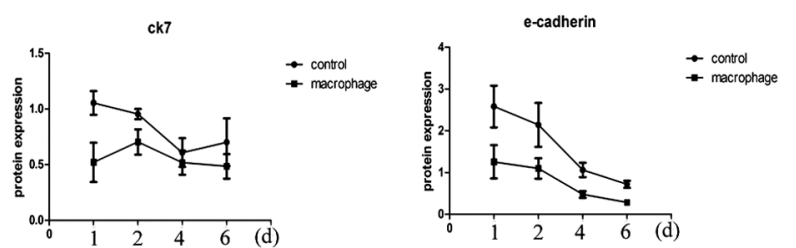

D
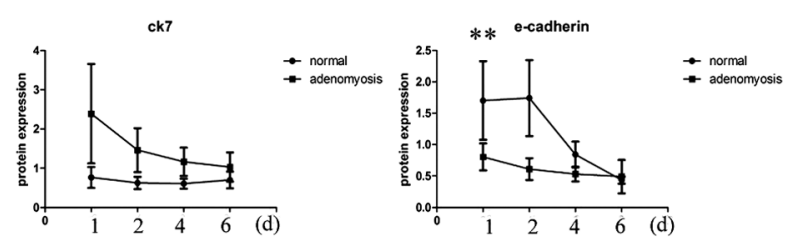

E
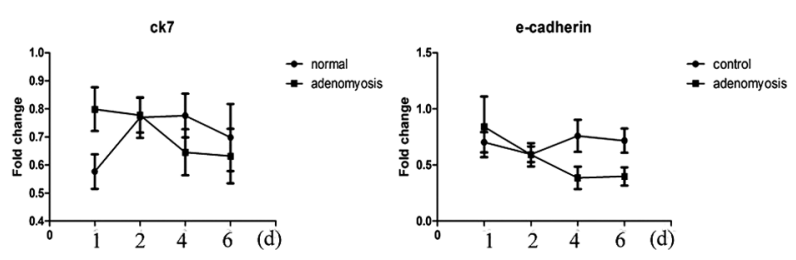
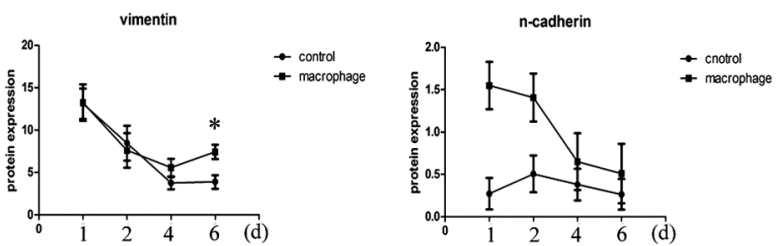

normal endometrium
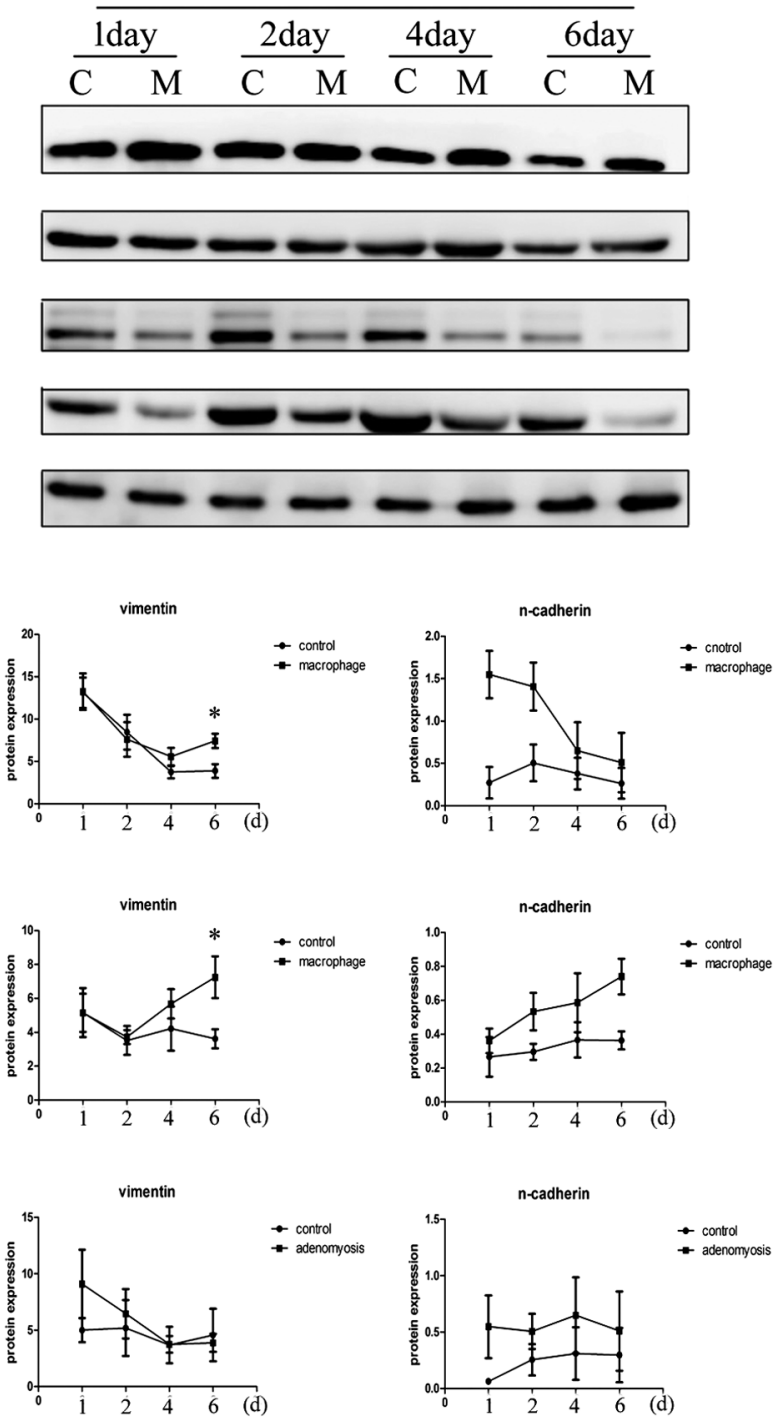

Figure 5 (A) Western blot of epithelial cells isolated from normal endometrium and adenomyosis eutopic endometrium. C, control group; $\mathrm{M}$, macrophages (co-culturing) group. (B) Statistical analysis of the protein expression levels of E-cadherin, CK7, N-cadherin and vimentin in eutopic epithelial cells between the control and co-culturing groups (Wilcoxon matched pairs test). (C) Statistical analysis of the protein expression levels of E-cadherin, CK7, N-cadherin and vimentin in normal epithelial cells between the control and co-culturing groups (Wilcoxon matched pairs test). (D) Statistical analysis of the protein expression levels of E-cadherin, CK7, N-cadherin and vimentin between normal and eutopic epithelial cells without co-culturing. (E) Statistical analysis of the fold changes of the protein expression levels between normal and eutopic epithelial cells after co-culturing. ${ }^{*} P<0.05 ;{ }^{*} P<0.01 ;{ }^{* * *} P<0.001$. The results are expressed as the mean \pm S.E.M.

the EMT process in adenomyosis research should be supplemented. The analysis of IHC did not find the definite evidence of EMT in the eutopic endometrium of adenomyosis in proliferative phage. However we found an EMT phenomenon in eutopic endometrium at secretory phase, and the expression levels of TGFB1 and SMAD3 that indicate signal pathway of EMT were higher in secretory phase, which meant that adenomyosis eutopic endometrium epithelial cells had been abnormally activated and dysfunctional. Through the 
A

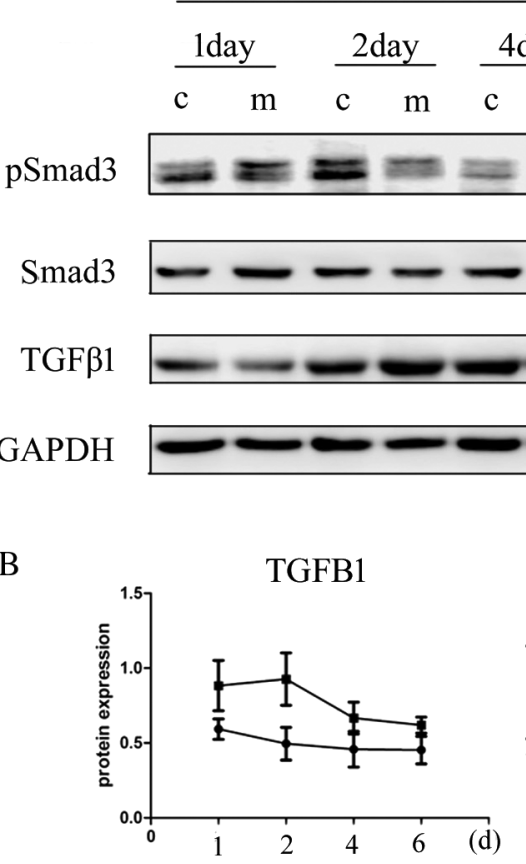

C

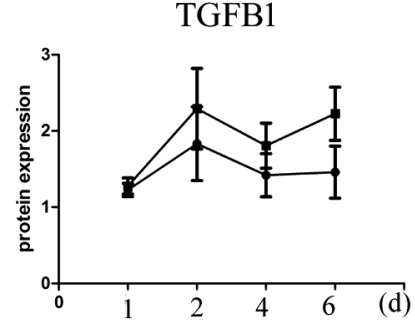

D

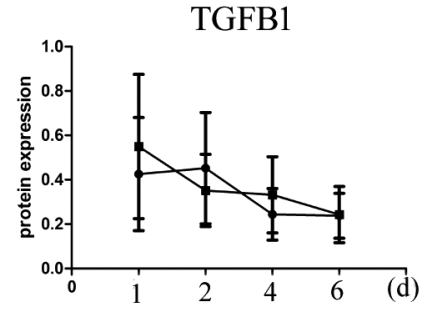

E

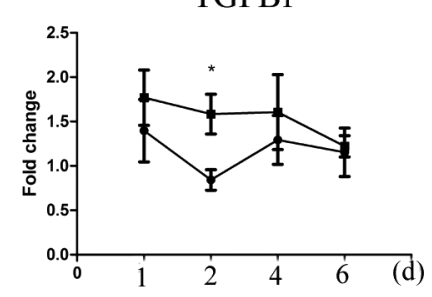

SMAD3
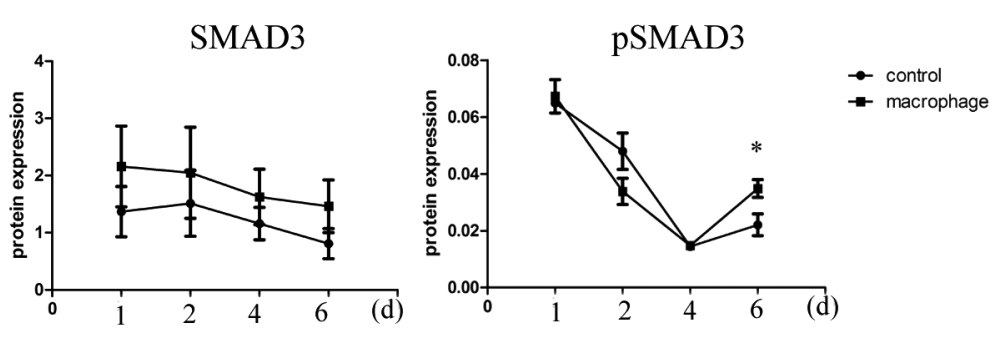

SMAD3
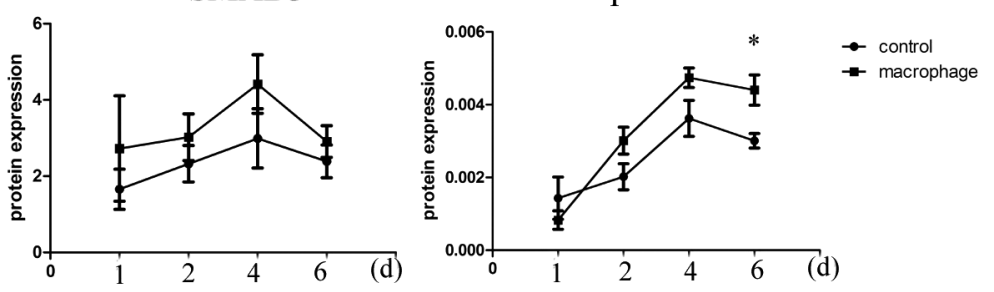

SMAD3
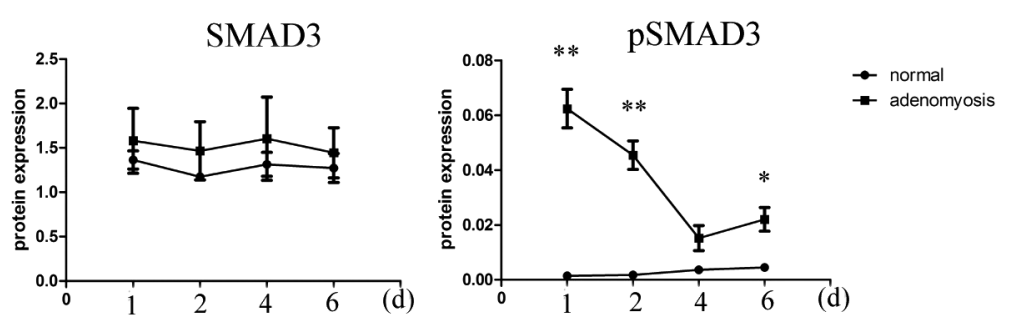

SMAD3

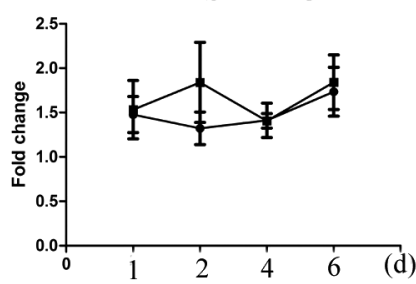

pSMAD3

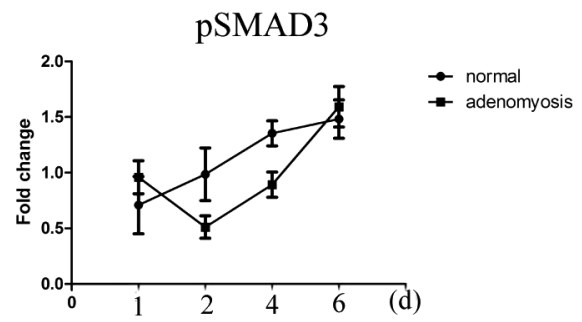

normal endometrium

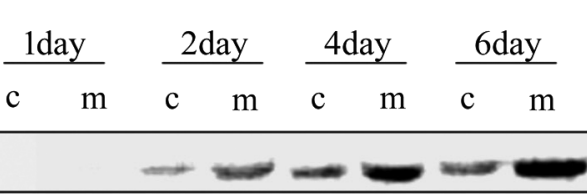

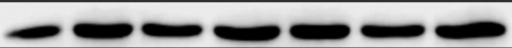

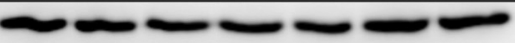

Figure 6 (A) Western blot of epithelial cells isolated from normal endometrium and eutopic endometrium of adenomyosis. C, control group; M, macrophages (co-culturing) group. (B) Statistical analysis of the protein expression levels of TGFB1 and SMAD3/pSMAD3 in eutopic epithelial cells between the control and co-culturing groups. (C) Statistical analysis of the protein expression levels of TGFB1 and SMAD3/ pSMAD3 in normal epithelial cells between the control and co-culturing groups. (D) Statistical analysis of the protein expression levels of TGFB1 and SMAD3/pSMAD3 between normal and eutopic epithelial cells without co-culturing. (E) Statistical analysis of the fold changes of the protein expression levels between normal and eutopic epithelial cells after co-culturing. ${ }^{*} P<0.05 ;{ }^{* *} P<0.01 ;{ }^{* * *} P<0.001$. The results are expressed as the mean \pm S.E.M. 
A

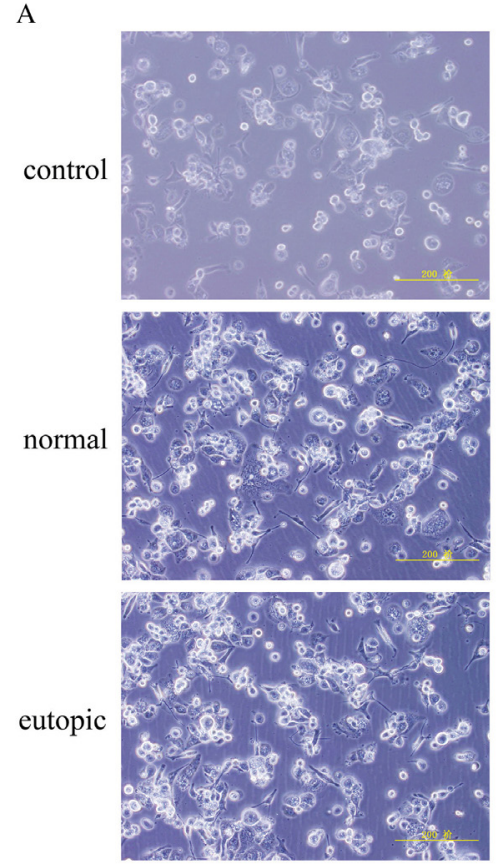

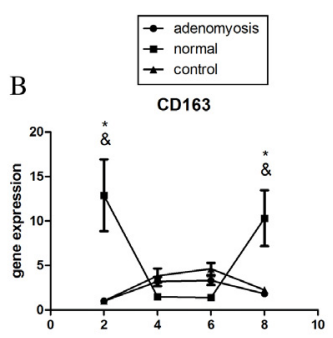
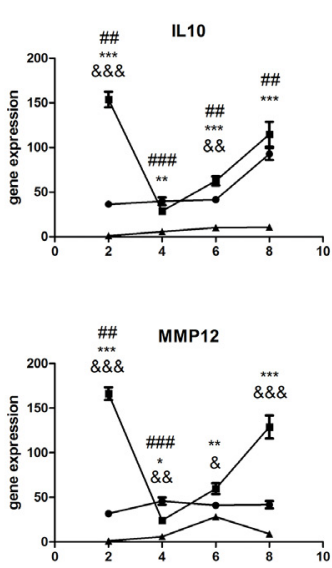

Figure 7 (A) Representative photomicrographs of macrophages with and without co-culturing (original magnification, $\times 400$ ). (B) Gene expression of THP-1-derived macrophages co-cultured with or without normal or eutopic epithelial cells. ${ }^{*}$ Normal group vs control group; \#adenomyosis group vs control group; ${ }^{\text {normal group vs }}$ adenomyosis group. ${ }^{* / \# / \&} P<0.05 ;{ }^{* * / \# \# / \& \& P} P<0.01 ;{ }^{* * * / \# \# / \& \& \& P<0.001}$. The results are expressed as the mean \pm S.E.M.

comparison during the menstrual cycle, we found that the pathway of TGFB1/SMAD3 (pSMAD3) was activated in the secretory phage, and the expression of E-cadherin was downregulated. These phenomena were consistent with previous research (Kim et al. 2005, Chen et al. 2010). We drew two reasons for the upregulated TGFB1/SMAD3 (pSMAD3) pathway in secretory phase of eutopic endometrium: one is progesterone, as a previous study described that the progesterone induces stromal decidualization indirectly by enhancing the expression and secretion of TGFB1 from epithelial cells (Kim et al. 2005); the other is the microenvironment, which stimulated the pathway to lead to EMT.

Recently, the growing body of literature strongly suggested that macrophages play a critical role in EMT regulation (Liu et al. 2013, Fan et al. 2014, Su et al. 2014), but its role has not been clarified in adenomyosis yet. In this study, primary epithelial cells were induced by THP1-derived macrophages to undergo EMT. In order to keep uniformity, we chose THP-1-derived macrophages which were the factor that induced EMT in epithelial cells (Dehai et al. 2014, Yang et al. 2016) to induce EMT process of normal and eutopic epithelial cells. The EMT process of epithelial cells was determined by the exact amount of exposure of THP-1-derived macrophages

compared with the controls. Eutopic and normal epithelial cells underwent EMT. Immunocytochemical analyses of CK7 and vimentin showed the cellular morphology. Although the changes of cell morphology were less obvious than protein expression, some epithelial cells have typical mesenchymal-like morphology, which was consistent with our previous study on the topic of EMT process of Ishikawa cells (Min et al. 2017). Statistical analysis of western blot showed that the changes of E-cadherin and CK7 were evident at some time points, but the upregulation of $\mathrm{N}$-cadherin and vimentin was not obvious in this study. The EMT of normal epithelial cells demonstrated that the microenvironment and macrophages played important roles in the EMT of adenomyosis. Considerable research has clarified the involvement of macrophages in the adenomyosis process (Yang et al. 2006, Khan et al. 2010, Tremellen \& Russell 2012). However the present study proved, from another perspective, that macrophages were indispensable in the pathogenesis of adenomyosis.

After comparing the eutopic and normal epithelial cells without co-culturing, changes in the expression of E-cadherin, CK7, N-cadherin and vimentin in epithelial cells were compared between the eutopic and normal groups after co-culturing with macrophages. Consistent with a recent study (Xiong et al. 2016), no significant difference in the expression levels of E-cadherin and $\mathrm{N}$-cadherin was observed between normal and eutopic epithelial cells. Moreover, no significant difference in the expression levels of CK7 and vimentin was observed between the two groups. In addition, after co-culturing with macrophages, the fold changes of downregulated E-cadherin and CK7, and upregulated $\mathrm{N}$-cadherin and vimentin have no difference between the eutopic endometrium group and the normal endometrium group. So, we speculate that macrophages can equally induce EMT process in eutopic and the normal epithelial cells.

The TGFB1/SMAD3 signaling pathway is essential for the EMT process. Although the upregulation of TGFB1 and SMAD3 was statistically insignificant, the protein expression levels of pSMAD3 were higher in the co-cultured epithelial cells than in the control. The expression levels of TGFB1, SMAD3 and pSMAD3 in epithelial cells were compared between eutopic and normal epithelial cells without co-culturing with macrophages. Then, the expression changes of TGFB1, SMAD3 and pSMAD3 in epithelial cells were compared between eutopic and normal groups after co-culturing with macrophages. The protein expression levels of TGFB1 and SMAD3 between eutopic and normal endometrium groups differed insignificantly, which is inconsistent with previous studies (Johnson et al. 2005, Cruz et al. 2015). Another research reported that only weak upregulation of TGFB1 in endometriosis eutopic endometrium occurred compared with the controls (Goteri et al. 2015). The present study is the first to compare the protein expression levels of TGFB1 and 
SMAD3 between adenomyotic eutopic and normal endometria, and the authors hope that this study could gain more attention from researchers involved in mechanism studies of adenomyosis. No significant difference in protein expression was observed between eutopic and normal endometrium groups without co-culturing; it was the same with the fold changes of TGFB1 and SMAD3 after co-culturing with macrophages in the eutopic endometrium group than in the normal endometrium group. This result was not consistent with our IHC analyses, and we speculated that the lack of steroids in the in vitro culture medium influenced it. However it is worth noting that the expressions of pSMAD3 were upregulated both in normal and eutopic epithelial cells after co-culture. But the fold changes of pSMAD3 after co-culturing have no significance. Thus, it is proved that the stimulation and induction of EMT process in the epithelial cells of the adenomyotic eutopic endometrium and normal endometrium is parallel.

Abnormal levels of immunecells, such as macrophages, have been largely reported in adenomyosis (Ota et al. 1998, Zhihong et al. 2016). Increased knowledge on the immune aspects of the pathogenesis of adenomyosis may allow the development of novel medical therapies for this debilitating condition. To our knowledge, macrophages, which play important roles in innate and acquired immunity, together with natural killer cells and cytotoxic T-lymphocytes in healthy women, can destroy misplaced endometrial cells (Dmowski et al. 1998). However, during the past decades, several reports identified a decrease or impairment in the cellmediated immunity of women with adenomyosis or endometriosis (Matarese et al. 2003, Guo et al. 2016). In this study, epithelial cells from normal and eutopic endometria can induce macrophages to polarize to the M2 type. However the inducing capability of the normal endometrium group was more intense than that of the eutopic endometrium group. CD163 was a hallmark of M2 macrophage. In our study, the expression of it was not higher in THP-1-derived macrophages after co-culture with eutopic epithelial cells. That signifies that the ratios of M2 to pan-macrophages were significantly lower in adenomyosis patients than those in normal group. It can be consistent with a previous study performed in endometriosis (Takebayashi et al. 2015). Khan et al. demonstrated that, compared to control women without endometriosis, women with endometriosis had Escherichia coli contamination in menstrual blood with increased levels of bacterial endotoxin in the menstrual fluid and peritoneal fluid (Berbic et al. 2009); this will affect the polarization of macrophages. Thus, we suspect that this phenomenon exists in adenomyosis as well. On the other hand, adenomyosis itself might be the cause of the incapable polarization of M2 macrophages in the eutopic endometrium. Given the failure of polarization to $M 2$ in the eutopic endometrium group, the misplaced endometrial cells might escape macrophage's engulfment and elimination. This incapacity can result from the eutopic endometrium itself or pelvic microenvironment. As the uterine cavity is connected to the pelvic cavity through the oviducts, some substances like immune cells in the pelvic cavity can flow into the uterine cavity, especially during the process of ovulation. Moreover, fluctuations in the genetic levels of CD163, IL10 and MMP12 were observed in the normal endometrium group because of the sensitive characteristics and homeostasis of macrophages. The weak activation of macrophages in the eutopic endometrium group demonstrated that the eutopic endometrium was immunotolerant.

Inconclusion, endometrialcellsandmicroenvironment are two important factors in the pathogenesis of adenomyosis. This study showed that both epithelial cells isolated from eutopic and normal endometria can be equally induced by macrophages to undergo EMT. The polarization of macrophages to M2 was less intense in the eutopic endometrium group than in the normal endometrium group. Further study is required to examine the immunity mechanism of the incapability, even failure, of polarization to $\mathrm{M} 2$ of inducing macrophages in eutopic endometrium of adenomyosis. Because, in eutopic epithelial cells, the expression levels of E-cadherin and pSMAD3 were higher than normal; we can attribute the incapable polarization to $\mathrm{M} 2$ to it. Our current findings suggest that adenomyosis may be promoted by the ability of epithelial cells derived from the eutopic endometrium to undergo EMT and the incapability of inducing macrophages to polarize to M2. Thus, immune regulation and inflammation reaction reduction may help relieve adenomyosis.

\section{Declaration of interest}

The authors declare that there is no conflict of interest that could be perceived as prejudicing the impartiality of the research reported.

\section{Funding}

This work was supported by the National Science Foundation of China (grant number 81571417) and the Technology Development Plan of Shandong Province (grant number 2015GSF118092).

\section{Authors' contribution statement}

Guoyun Wang and Dong Li conceived and designed the study. Min An analyzed and interpreted data, and drafted the manuscript. Min An and Ming Yuan performed the experiments. Qiuju Li recruited patients and secured tissue samples. Every author participated in the writing and revision of the manuscript. 


\section{Acknowledgements}

We thank Prof. Chengjiang, Gao (Shandong University, School of Medicine, Department of Immunology, Jinan, Shandong, China) for providing the THP-1 cells as a gift to us. We also thank all the patients for agreeing to participate in our study.

\section{References}

Alcorn D, Maric C \& McCausland J 1999 Development of the renal interstitium. Pediatric Nephrology 13 347-354. (doi:10.1007/ s004670050624)

Benagiano G, Brosens I \& Habiba M 2013 Structural and molecular features of the endomyometrium in endometriosis and adenomyosis. Human Reproduction Update 20 386-402. (doi:10.1093/humupd/dmt052)

Berbic M, Schulke L, Markham R, Tokushige N, Russell P \& Fraser IS 2009 Macrophage expression in endometrium of women with and without endometriosis. Human Reproduction 24 325-332. (doi:10.1093/ humrep/den393)

Bergeron C, Amant F \& Ferenczy A 2006 Pathology and physiopathology of adenomyosis. Best Practice and Research Clinical Obstetrics and Gynaecology 20 511-521. (doi:10.1016/j.bpobgyn.2006.01.016)

Bird CC, McElin TW \& Manalo-Estrella P 1972 The elusive adenomyosis of the uterus - revisited. American Journal of Obstetrics and Gynecology 112 583-593. (doi:10.1016/0002-9378(72)90781-8)

Chan RWS, Schwab KE \& Gargett CE 2004 Clonogenicity of human endometrial epithelial and stromal cellss. Biology of Reproduction $\mathbf{7 0}$ 1738-1750. (doi:10.1095/biolreprod.103.024109)

Chen JJ, Yao PL, Yuan A, Hong TM, Shun CT, Kuo ML, Lee YC \& Yang PC 2003 Up-regulation of tumor interleukin-8 expression by infiltrating macrophages: its correlation with tumor angiogenesis and patient survival in non-small cell lung cancer. Clinical Cancer Research 9 $729-737$.

Chen Y-J, Li H-Y, Huang C-H, Twu N-F, Yen M-S, Wang P-H, Chou T-Y, Liu Y-N, Chao K-C \& Yang M-H 2010 Oestrogen-induced epithelialmesenchymal transition of endometrial epithelial cells contributes to the development of adenomyosis. Journal of Pathology 222 261-270. (doi:10.1002/path.2761)

Cruz CD, Del Puerto HL, Rocha AL, Cavallo IK, Clarizia AD, Petraglia F \& Reis FM 2015 Expression of Nodal, Cripto, SMAD3, phosphorylated SMAD3, and SMAD4 in the proliferative endometrium of women with endometriosis. Reproductive Sciences 22 527-533. (doi:10.1177/1933719114549855)

Dehai C, Bo P, Qiang T, Lihua S, Fang L, Shi J, Jingyan C, Yan Y, Guangbin W \& Zhenjun Y 2014 Enhanced invasion of lung adenocarcinoma cells after co-culture with THP-1-derived macrophages via the induction of EMT by IL-6. Immunology Letters 160 1-10. (doi:10.1016/j.imlet.2014.03.004)

Dmowski WP, Gebel H \& Braun DP 1998 Decreased apoptosis and sensitivity to macrophage mediated cytolysis of endometrial cells in endometriosis. Human Reproduction Update 4 696-701. (doi:10.1093/ humupd/4.5.696)

Fan QM, Jing YY, Yu GF, Kou XR, Ye F, Gao L, Li R, Zhao QD, Yang Y, Lu ZH et al. 2014 Tumor-associated macrophages promote cancer stem celllike properties via transforming growth factor-beta1-induced epithelialmesenchymal transition in hepatocellular carcinoma. Cancer Letters 352 160-168. (doi:10.1016/j.canlet.2014.05.008)

Ferenczy A 1998 Pathophysiology of adenomyosis. Human Reproduction Update 4 312-322. (doi:10.1093/humupd/4.4.312)

Galdiero MR, Garlanda C, Jaillon S, Marone G \& Mantovani A 2013 Tumor associated macrophages and neutrophils in tumor progression. Journal of Cellular Physiology 228 1404-1412. (doi:10.1002/jcp.24260)

Goteri G, Altobelli E, Tossetta G, Zizzi A, Avellini C, Licini C, Lorenzi T, Castellucci M, Ciavattini A \& Marzioni D 2015 High temperature requirement $A 1$, transforming growth factor beta1, phosphoSMAD2 and $\mathrm{Ki} 67$ in eutopic and ectopic endometrium of women with endometriosis. European Journal of Histochemistry 59 2570. (doi:10.4081/ ejh.2015.2570)

Guo J, Chen L, Luo N, Li C, Chen R, Qu X, Liu M, Kang L \& Cheng Z 2016 LPS/TLR4-mediated stromal cells acquire an invasive phenotype and are implicated in the pathogenesis of adenomyosis. Scientific Reports $\mathbf{6}$ 21416. (doi:10.1038/srep21416)

Johnson MC, Torres M, Alves A, Bacallao K, Fuentes A, Vega M \& Boric MA 2005 Augmented cell survival in eutopic endometrium from women with endometriosis: expression of c-myc, TGF-beta1 and bax genes. Reproductive Biology and Endocrinology 3 45. (doi:10.1186/14777827-3-45)

Kao A-P, Wang K-H, Chang C-C, Lee J-N, Long C-Y, Chen H-S, Tsai C-F, Hsieh T-H \& Tsai E-M 2011 Comparative study of human eutopic and ectopic endometrial mesenchymal stem cells and the development of an in vivo endometriotic invasion model. Fertility and Sterility 951308. e1301-1315.e1301. (doi:10.1016/j.fertnstert.2010.09.064)

Khan KN, Kitajima M, Hiraki K, Fujishita A, Sekine I, Ishimaru T \& Masuzaki H 2010 Changes in tissue inflammation, angiogenesis and apoptosis in endometriosis, adenomyosis and uterine myoma after GnRH agonist therapy. Human Reproduction 25 642-653. (doi:10.1093/humrep/ dep437)

Khan KN, Kitajima M, Hiraki K, Fujishita A, Nakashima M \& Masuzaki H 2015 Involvement of hepatocyte growth factor-induced epithelialmesenchymal transition in human adenomyosis. Biology of Reproduction 9235 .(doi:10.1095/biolreprod.114.124891)

Kim MR, Park DW, Lee JH, Choi DS, Hwang KJ, Ryu HS \& Min CK 2005 Progesterone-dependent release of transforming growth factor-beta1 from epithelial cells enhances the endometrial decidualization by turning on the SMAD signalling in stromal cells. Molecular Human Reproduction 11 801-808. (doi:10.1093/molehr/gah240)

Kunz G, Beil D, Huppert P \& Leyendecker G 2000 Structural abnormalities of the uterine wall in women with endometriosis and infertility visualized by vaginal sonography and magnetic resonance imaging. Human Reproduction 15 76-82. (doi:10.1093/humrep/15.1.76)

Kunz G, Beil D, Huppert P, Noe M, Kissler S \& Leyendecker G 2005 Adenomyosis in endometriosis - prevalence and impact on fertility. Evidence from magnetic resonance imaging. Human Reproduction 20 2309-2316. (doi:10.1093/humrep/dei021)

Lamouille S, Xu J \& Derynck R 2014 Molecular mechanisms of epithelialmesenchymal transition. Nature Reviews Molecular Cell Biology 15 178-196. (doi:10.1038/nrm3758)

Liu CY, Xu JY, Shi XY, Huang W, Ruan TY, Xie P \& Ding JL 2013 M2polarized tumor-associated macrophages promoted epithelialmesenchymal transition in pancreatic cancer cells, partially through TLR4/IL10 signaling pathway. Laboratory Investigation 93 844-854. (doi:10.1038/labinvest.2013.69)

Matarese G, De Placido G, Nikas Y \& Alviggi C 2003 Pathogenesis of endometriosis: natural immunity dysfunction or autoimmune disease? Trends in Molecular Medicine 9 223-228. (doi:10.1016/S14714914(03)00051-0)

Matsuzaki S \& Darcha C 2012 Epithelial to mesenchymal transition-like and mesenchymal to epithelial transition-like processes might be involved in the pathogenesis of pelvic endometriosis. Human Reproduction 27 712-721. (doi:10.1093/humrep/der442)

Min A, Dong L, Ming Y, Qiuju L, Lu Z \& Guoyun W 2017 Interaction of macrophages and endometrial cells induces epithelial-mesenchymal transition-like processes in adenomyosis. Biology of Reproduction 96 46-57. (doi:10.1095/biolreprod.116.144071)

Ota H, Igarashi S, Hatazawa J \& Tanaka T 1998 Is adenomyosis an immune disease? Human Reproduction Update 4 360-367. (doi:10.1093/ humupd/4.4.360)

Scotton CJ \& Chambers RC 2007 Molecular targets in pulmonary fibrosis: the myofibroblast in focus. Chest 132 1311-1321. (doi:10.1378/ chest.06-2568)

Shao J, Zhang B, Yu JJ, Wei CY, Zhou WJ, Chang KK, Yang HL, Jin LP, Zhu XY \& Li MQ 2016 Macrophages promote the growth and invasion of ESCs by down-regulating IL-24 in EMS. Reproduction 152 673-682. (doi:10.1530/REP-16-0278)

Shen M, Liu X, Zhang H \& Guo S-W 2015 Transforming growth factor $\beta 1$ signaling coincides with epithelial-mesenchymal transition and fibroblast-to-myofibroblast transdifferentiation in the development of adenomyosis in mice. Human Reproduction 31 355-369. (doi:10.1093/ humrep/dev314)

Su S, Liu Q, Chen J, Chen J, Chen F, He C, Huang D, Wu W, Lin L, Huang W et al. 2014 A positive feedback loop between mesenchymal-like 
cancer cells and macrophages is essential to breast cancer metastasis. Cancer Cell 25 605-620. (doi:10.1016/j.ccr.2014.03.021)

Takebayashi A, Kimura F, Kishi Y, Ishida M, Takahashi A, Yamanaka A, Wu D, Zheng L, Takahashi K, Suginami H et al. 2015 Subpopulations of macrophages within eutopic endometrium of endometriosis patients. American Journal of Reproductive Immunology 73 221-231. (doi:10.1111/aji.12331)

Tremellen KP \& Russell P 2012 The distribution of immune cells and macrophages in the endometrium of women with recurrent reproductive failure. II: adenomyosis and macrophages. Journal of Reproductive Immunology 93 58-63. (doi:10.1016/j.jri.2011.12.001)

Wheelock MJ, Shintani Y, Maeda M, Fukumoto Y \& Johnson KR 2008 Cadherin switching. Journal of Cell Science 121 727-735. (doi:10.1242/ jcs.000455)

Xiong Y, Liu Y, Xiong W, Zhang L, Liu H, Du Y \& Li N 2016 Hypoxia-inducible factor 1 alpha-induced epithelial-mesenchymal transition of endometrial epithelial cells may contribute to the development of endometriosis. Human Reproduction 31 1327-1338. (doi:10.1093/humrep/dew081)

Xue J, Zhang H, Liu W, Liu M, Shi M, Wen Z \& Li C 2013 Metformin inhibits growth of eutopic stromal cells from adenomyotic endometrium via AMPK activation and subsequent inhibition of AKT phosphorylation: a possible role in the treatment of adenomyosis. Reproduction $\mathbf{1 4 6}$ 397-406. (doi:10.1530/REP-13-0135)
Yang JH, Wu MY, Chang DY, Chang CH, Yang YS \& Ho HN 2006 Increased interleukin-6 messenger RNA expression in macrophagecocultured endometrial stromal cells in adenomyosis. American Journal of Reproductive Immunology 55 181-187. (doi:10.1111/j.16000897.2005.00363.x)

Yang M, Ma B, Shao H, Clark AM \& Wells A 2016 Macrophage phenotypic subtypes diametrically regulate epithelial-mesenchymal plasticity in breast cancer cells. BMC Cancer 16 419. (doi:10.1186/s12885-0162411-1)

Zhihong N, Yun F, Pinggui Z, Sulian Z \& Zhang AJ 2016 Cytokine profiling in the eutopic endometrium of adenomyosis during the implantation window after ovarian stimulation. Reproductive Sciences 23 124-133. (doi:10.1177/1933719115597761)

Received 24 March 2017

First decision 13 April 2017

Revised manuscript received 7 May 2017

Accepted 11 May 2017 